\title{
Some Effects on Relativistic Quantum Systems Due to a Weak Gravitational Field
}

\author{
Geusa de A. Marques ${ }^{1}$, Sandro G. Fernandes ${ }^{2}$, and V. B. Bezerra ${ }^{3}$ \\ 1. Departamento de Física, Universidade Federal de \\ Campina Grande, 58109-790, Campina Grande, Pb, Brazil \\ 2. Departamento de Matemática, Fundação Universidade \\ Estadual de Alagoas, 57601-460, \\ Palmeira dos Índios, Al, Brazil. \\ 3. Departamento de Física, Universidade Federal da Paraíba, \\ 58051-970, João Pessoa, Pb, Brazil
}

(Received on 14 October, 2005)

\begin{abstract}
We study the behaviour of relativistic quantum particles in the space-time generated by a moving mass current, in the weak field approximation. We solve the Dirac equation in this gravitational field and calculate the current associated with the particles.
\end{abstract}

The study of the behaviour of quantum systems under the influence of curved space-times goes back to the end of the 1920 s and to the beginning of the 1930s[1], when the generalization of the Schrödinger and Dirac equations to curved spaces has been discussed, motivated by the idea of constructing a theory combining quantum physics and general relativity. Along this line of research the hydrogen atom has been studied in particular curved space-times[2, 3]. These investigations showed that the energy levels of an atom placed in a gravitational field is shifted as a result of the interaction of the atom with the space-time curvature[3]-[5]. This shift in the energy of each atomic level would depend on the features of the space-time.

The general theory of relativity, as a metric theory, predicts that gravitation is manifested as the curvature of space-time. Therefore, it is of interest to know how the curvature of spacetime at the position of the atom affects its spectrum. On the other hand, we know that there are situations in which particles are constrained to move in a region where the Riemann curvature tensor vanishes and even in this case they exhibit gravitational effects arising from a region of non-zero curvature from which they are excluded[6]. In a more general sense, we have the case in which particles are constrained to move in a region where the Riemann curvature tensor does not vanish but does depend on certain parameter of the metric such as the velocity or the angular momentum of the source. In this case we have effects on the system associated with parameters which do not influence the curvature of the space-time as we will see.

In what follows we present the study concerning the behaviour of a relativistic particle placed in the gravitational field generated by a cylindrical distribution of matter with uniform density along the $z$-axis moving slowly, whose metric reads[7]

$$
\begin{aligned}
d s^{2}= & -(1-\Phi(\rho)) d t^{2}+(1+\Phi(\rho)) \\
& \times\left(d \rho^{2}+\rho^{2} d \varphi^{2}+d z^{2}\right)-4 v \Phi(\rho) d z d t,
\end{aligned}
$$

where $\Phi(\rho)$ represents the Newtonian potential produced by this source and satisfies the condition $\Phi(\rho)^{2} \approx 0$, in the weak field approximation and $v$ is the velocity of the distribution of matter. This quantity also satisfies the condition $v^{2} \approx 0$. This metric is characterized by two parameters, namely, the mass of the source and its velocity. It is interesting to call attention to the fact that in the weak field approximation, the Riemann curvature tensor outside the cylindrical source is completely determined by the Newtonian potential.

For this space-time, the curvature outside the distribution of matter does not depend on its velocity, in the weak field approximation. This means that for the weak gravitational field associated with slowly moving mass currents, the local effects of the curvature associated with the velocity of the source are absent outside it.

The covariant Dirac equation in a curved space-time, for a massive spinor field $\Psi$ is given by

$$
\left[i \gamma^{\mu}(x) \partial_{\mu}-i \gamma^{\mu}(x) \Gamma_{\mu}(x)-m\right] \Psi(x)=0
$$

where $\gamma^{\mu}(x)$ are the generalized Dirac matrices and are given in terms of the standard flat space Dirac matrices $\gamma^{(a)}$ as

$$
\gamma^{\mu}(x)=e_{(a)}^{\mu}(x) \gamma^{(a)}
$$

where $e_{(a)}^{\mu}(x)$ are the tetrads components defined by $e_{(a)}^{\mu}(x) e_{(b)}^{v}(x) \eta^{(a)(b)}=g^{\mu v}$, where the Greek indices are connected with the tensor world indices(coordinate basis system) and the Latin indices denote Lorentz indices which are connected with the local Minkowski coordinate system(tetrads).

The probability current can be written using the Gordon decomposition

$$
\begin{aligned}
j^{\mu}= & \frac{1}{2 m} \partial_{\mu}\left(\bar{\Psi} \sigma^{\mu \lambda} \Psi\right)+\frac{i}{4 m} g^{\mu \lambda}\left[\bar{\Psi} \partial_{\lambda} \Psi-\left(\partial_{\lambda} \bar{\Psi}\right) \Psi\right] \\
& +\frac{i}{4 m} \bar{\Psi}\left(\left[\partial_{\lambda} \gamma^{\lambda}, \gamma^{\mu}\right]+\left[\gamma^{\lambda}, \partial_{\lambda} \gamma^{\mu}\right]\right) \Psi \\
& +\frac{i}{2 m} \bar{\Psi}\left[\gamma^{\lambda} \Gamma_{\lambda}, \gamma^{\mu}\right] \Psi
\end{aligned}
$$

Now, we will consider a massive spinor particle in the weak gravitational field due to the distribution of matter we are considering. Thus, in order to solve the Dirac equation for a massive particle, given by Eq. (2), in this space-time, let us choose the following set of tetrads 


$$
e_{(a)}^{\mu}=\left(\begin{array}{llll}
1+\frac{\Phi(\rho)}{2} & 0 & 0 & -2 v \Phi(\rho) \\
0 & 1-\frac{\Phi(\rho)}{2} & 0 & 0 \\
0 & 0 & \frac{1-\frac{\Phi(\rho)}{2}}{\rho} & 0 \\
0 & 0 & 0 & 1-\frac{\Phi(\rho)}{2}
\end{array}\right) .
$$

In order to do the calculations, let us consider that the particle is restricted to move in a narrow region such that $\Phi(\rho)$ is approximately constant and equal to $\Phi_{1}$. Thus, we will get the following Dirac equation in the space-time of a moving mass current

$$
\begin{gathered}
\left\{i\left[\left(1+\frac{\Phi_{1}}{2}\right) \gamma^{(0)}-2 v \Phi_{1} \gamma^{(3)}\right] \frac{\partial}{\partial t}\right. \\
+i\left(1-\frac{\Phi_{1}}{2}\right) \gamma^{(1)} \frac{\partial}{\partial \rho}+\frac{i}{2 \rho}\left(1-\frac{\Phi_{1}}{2}\right) \gamma^{(2)} \frac{\partial}{\partial \varphi} \\
\left.+i\left(1-\frac{\Phi_{1}}{2}\right) \gamma^{(3)} \frac{\partial}{\partial z}+\frac{i}{2 \rho}\left(1+\frac{\Phi_{1}}{2}\right) \gamma^{(1)}-m\right\} \Psi=0 .
\end{gathered}
$$

Due to the fact that the space-time is static and symmetric under translations along the $z$-axis and rotations around this axis, the solution of Eq. (1) can be written as

$$
\Psi_{j}=e^{-i E t} e^{i(l \varphi+k z)} R_{j}(\rho) \quad, \quad j=1,2,3,4
$$

where

$$
\begin{aligned}
R_{1}(\rho)= & R_{3}(\rho)=C_{1} \rho^{\left(\frac{1}{2}-\frac{u}{2 B}\right)} J_{\sigma}\left(\sqrt{\frac{\tau}{B}} \rho\right) \\
& +C_{2} \rho^{\left(\frac{1}{2}-\frac{u}{2 B}\right)} N_{\sigma}\left(\sqrt{\frac{\tau}{B}} \rho\right)
\end{aligned}
$$

and

$$
\begin{aligned}
R_{2}(\rho)= & R_{4}(\rho)=C_{1}^{\prime} \rho^{\left(\frac{1}{2}-\frac{u}{2 B}\right)} J_{\bar{\sigma}}\left(\sqrt{\frac{\bar{\tau}}{B}} \rho\right) \\
& +C_{2}^{\prime} \rho^{\left(\frac{1}{2}-\frac{u}{2 B}\right)} N_{\bar{\sigma}}\left(\sqrt{\frac{\bar{\tau}}{B}} \rho\right)
\end{aligned}
$$

with

$$
\begin{aligned}
& \sigma=\frac{1}{2} \sqrt{\frac{B^{2}-2 u B+u^{2}-4 \omega^{2} B}{B^{2}}} ; \\
& \bar{\sigma}=\frac{1}{2} \sqrt{\frac{B^{2}-2 \bar{u} B+\bar{u}^{2}-4 \bar{\omega}^{2} B}{B^{2}}} ; \\
& \omega=B^{2}\left[l(l-1)+\frac{1}{4}-\frac{1}{2 B}\right] ; \\
& \tau=p q ; \quad \bar{u}=-B^{2}\left(\frac{1}{2}-l\right) ; \\
& u=B^{2}\left(l+\frac{1}{2}\right) ; \quad \bar{\omega}=-B^{2}\left(l+\frac{1}{4}\right) ; \\
& \bar{\tau}=(\bar{v} E+B k)^{2}-(A E+m)^{2},
\end{aligned}
$$

where $A=1+\frac{\Phi_{1}}{2}, B=1-\frac{\Phi_{1}}{2}$ and $\bar{v}=2 \nu \Phi_{1}$

Note that these solutions depend on the velocity of the source, as well as on the Newtonian potential. In this case the Riemann tensor does not depend on the velocity of the source, and therefore, the obtained result means that the dependence of the solution with the velocity is of purely global origin.

The current can be computed using Eq.(4), which is this case results in the following expressions for the components

$$
j_{t}=\vec{\nabla} \cdot \vec{P}-\frac{2 v}{\rho^{2}} M_{\varphi}+\rho_{\text {conv }},
$$

$$
\begin{gathered}
j_{\rho}=-\partial_{t} P_{\rho}+(\vec{\nabla} \times \vec{M})_{\rho}-\frac{2 v}{\rho} \partial_{t} M_{\rho}+j_{\rho, c o n v}, \\
j_{\varphi}=-\partial_{t} P_{\varphi}+(\vec{\nabla} \times \vec{M})_{\varphi}+j_{\varphi, c o n v} \\
j_{z}=-\partial_{t} P_{z}+(\vec{\nabla} \times \vec{M})_{z}-\frac{2 v}{\rho} \partial_{t} M_{\rho}+j_{z, c o n v} .
\end{gathered}
$$

With regard to an external electromagnetic field, $\vec{P}$ and $\vec{M}$ are the polarization and magnetization current densities, respectively. The components of the polarization vector are given by $P_{\rho}=\frac{i}{2 m} \bar{\Psi} \gamma_{(0)} \gamma_{(\rho)} \Psi, \quad P_{\varphi}=\frac{i}{2 m} \bar{\Psi} \gamma_{(0)} \gamma_{(\varphi)} \Psi, \quad P_{z}=$ $\frac{i}{2 m} \bar{\Psi} \gamma_{(0)} \gamma_{(z)} \Psi$

$$
\gamma_{\rho}=\gamma_{1} \cos \varphi+\gamma_{2} \sin \varphi \text { and } \gamma_{\varphi}=\gamma_{1} \sin \varphi+\gamma_{2} \cos \varphi
$$

The magnetization density vector has components given by the expressions $M_{\rho}=\frac{i}{4 m} \bar{\Psi}\left[\gamma_{(\varphi)}, \gamma_{(z)}\right] \Psi, \quad M_{\varphi}=$ $\frac{i}{4 m} \bar{\Psi}\left[\gamma_{(z)}, \gamma_{(\rho)}\right] \Psi, \quad M_{z}=\frac{i}{4 m} \bar{\Psi}\left[\gamma_{(\rho)}, \gamma_{(\varphi)}\right] \Psi$.

From the obtained results, we conclude that the solution of the Dirac equation, as well as the current associated with the particle depend on the velocity of the source. This is an example of a global phenomenon associated with this gravitational field.

It is worth calling attention to the fact that in the region of motion of the particle, the Riemann curvature, in the weak field approximation, does not depend on the velocity of the source. This result means that, even in the situation in which the particle is constrained to move in a region where the Riemann curvature does not depend on the velocity of the source, it exhibits a gravitational effect associated with this quantity. This dependence on a parameter which does not have any influence on the Riemann curvature tensor, in the weak field approximation, is a manifestation of a global phenomenon associated with these gravitational fields, called gravitational Aharonov-Bohm effect[7, 8].

\section{Acknowledgments}

We acknowledge Conselho Nacional de Desenvolvimento Científico e Tecnológico (CNPq) and Coordenação de 
Aperfeiçoamento de Pessoal de Nível Superior (CAPES) Programa PROCAD and FAPESQ-Pb/CNPq(PRONEX) for partial financial support.
[1] V. Fock, Z. Phys. 53, 592 (1928); E. Schrödinger, Physica 6, 899 (1932); W. Pauli, Ann. Phys. (Leipzig) 18, 337 (1933).

[2] J. Audretsch and G. Schäfer, Gen. Rel. Grav. 9 , 243 (1978); id. 9, 489 (1978) and references therein.

[3] L. Parker, Phys. Rev. Lett. 44,1559 (1980).

[4] L. Parker, Phys. Rev. D22, 1922 (1980); id. D 24, 535 (1981).

[5] L. Parker and L. O. Pimentel, Phys. Rev. D25, 3180 (1982).
[6] Geusa de A. Marques and Valdir B. Bezerra, Phys. Rev. D66, 105011 (2002); Class. Quantum Grav. 19, 985 (2002).

[7] J. K. Lawrence, D. Leiter, and G. Szamosi, Nuovo Cim. 178, 113 (1973).

[8] Geusa de A. Marques and V. B. Bezerra, Mod. Phys. Lett. A19, 49 (2004). 